\title{
Venous thromboembolism and sarcoidosis: co-incidence or coexistence?
}

\author{
ANNA GOLJAN-GEREMEK', MARCIN GEREMEK', ELZBIETA PUSCINSKA ${ }^{1}$, PAWEL SLIWINSKI \\ ${ }^{1} 2^{\text {nd }}$ Department of Respiratory Medicine, National Tuberculosis and Lung Diseases Research Institute, Warsaw, Poland \\ ${ }^{2}$ Department of Vascular Surgery, Wolski Hospital, Warsaw, Poland \\ ${ }^{3} 4^{\text {th }}$ Department of Respiratory Medicine, National Tuberculosis and Lung Diseases Research Institute, Warsaw, Poland
}

\begin{abstract}
The association between venous thromboembolism (VTE) and sarcoidosis has been reported recently, nevertheless the true incidence of co-incident sarcoidosis and VTE is unknown. Sarcoidosis as a chronic disease of immune dysregulation might be associated with an increased risk of VTE. The mechanisms responsible for VTE development are not clear and may be influenced by several factors: activity of inflammation, clinical characteristics of sarcoidosis and comorbidities. Pulmonary embolism $(P E)$ as a potentially fatal condition should be considered in all of the patients with sarcoidosis in whom worsening of the respiratory status is diagnosed. A high plasma D-dimers $(D D)$ level may be suggestive of VTE, nevertheless elevated plasma DD should be interpreted with caution, in the context of the active inflammatory process. If sarcoidosis appears to be one of risk factors for VTE development, further investigations are needed to define the pro-thrombotic phenotype of this disease.
\end{abstract}

Key words: sarcoidosis, inflammation, venous thromboembolism.

(Cent Eur J Immunol 2015; 40 (4): 477-480)

\section{Introduction}

Sarcoidosis is a systemic disease of unknown etiology with systemic inflammation leading to formation of granulomas. Lung parenchyma and/or mediastinal lymph nodes are affected in $>90 \%$ of patients [1]. Macrophages and activated lymphocytes, an immunological hallmark of sarcoidosis, are also involved in the process of thrombin activation and fibrin formation [2, 3]. The association between sarcoidosis and venous thromboembolism (VTE) has been reported recently $[4,5]$, nevertheless it is still not clear if sarcoidosis is a risk factor for VTE. Thus, the aim of the present study was to describe possible mechanisms that could be responsible for the increased occurrence of VTE in sarcoidosis.

\section{Epidemiology}

A higher risk of pulmonary embolism (PE) in sarcoidosis patients has been reported recently, according to data from the UK, with a risk ratio of 2.0 (95\% CI: 1.1-3.4), in patients under 65 years of age, comparing to a reference cohort [4]. Swigris et al. found PE in $2.5 \%$ of US decedents with sarcoidosis, regardless of gender, race or age [5]. In the retrospective analysis of clinical outcome and comorbidities among 798 sarcoidosis patients who have been hospitalized in the $2^{\text {nd }}$ Pulmonary Department of our
Institution between 2004 and 2014, 8 symptomatic cases of PE (one fatal case), were found.

\section{Possible mechanisms involved}

The mechanisms that could predispose sarcoidosis patients to the development of VTE may be related to several factors: activity of inflammation, clinical picture of sarcoidosis or comorbidities.

\section{Inflammatory profile}

A close anatomic relation between the site of active sarcoidosis and thrombus formation was described in the literature: mural thrombus in myocardial sarcoidosis [6], cerebral vein thrombosis in neurosarcoidosis [7-9], thoracic vein thrombosis in mediastinal sarcoidosis [10-12], portal vein hypertension in hepatic sarcoidosis [13], suggesting the local hypercoagulability status as the cause of thrombosis. Local hypercoagulability might have been the consequence of active granulomatous inflammation with an increased concentration of inflammatory markers [1419]. We have found recently features of disease activity: elevated CRP, ESR, and ACE, monocytosis and hypergammaglobulinemia in our patients with sarcoidosis who developed VTE (unpublished data). Nonspecific hypergammaglobulinemia reflecting the $\mathrm{B}$ cell activation, which is

Correspondence: Anna Goljan-Geremek MD, PhD, $2^{\text {nd }}$ Department of Respiratory Medicine, National Tuberculosis and Lung Diseases Research Institute, Płocka 26, 01-138 Warsaw, Poland, e-mail: a.goljan@ gmail.com 
observed in the active disease may indicate a more generalized inflammatory process, although the definitive role for humoral immunity in the pathogenesis of sarcoidosis is not established [20].

The regulatory influence of cytokines produced by local mononuclear phagocytes results in granulomatous inflammation in sarcoidosis patients [3, 21, 22]. Cermak et al. demonstrated that peripheral blood monocytes incubated with highly purified ( $>90 \%$ ) human CRP for 6 hours present a significant increase in the tissue factor (TF) expression and acquire a significant pro-coagulant activity [23]. The same phenomenon might be responsible for the increased pro-coagulant ability of granulomatous inflammation in sarcoidosis. In sarcoidosis patients, as compared to normal control subjects, an enhanced activity of BAL fluid TF and plasma Factor VII were found [24]. The radiologic stage of the disease correlated with TF activity. Only patients with stage II or stage III disease had a consistently elevated procoagulant activity that corresponded to the site most involved radiographically [24].

Different cytokines modulate the risk for venous thrombosis [14]. Shorr et al. found that DD plasma levels correlate with activation of the proinflammatory cytokine cascade [interleukin (IL)-6, IL-8, tumor necrosis factor $\alpha$ (TNF- $\alpha$ ) but not with IL-10 [25]. Tumor necrosis factor $\alpha$ (TNF- $\alpha$ ) is a key cytokine in the pathogenesis of sarcoidosis and may indicate the activity of the disease [26, 27]. Elevated TNF- $\alpha$ concentration in active sarcoidosis might be responsible for the increased risk of VTE. Locally derived IL-6 and IL- 8 were increased in sarcoidosis and correlated with activity of this granulomatous lung disease [19]. Interleukin 10, an anti-inflammatory cytokine, expresses protective potential against venous thrombosis $[14,28]$. A down-modulating mechanism resulting in the spontaneous resolution of sarcoid alveolitis is partially controlled by an increased local secretion of IL-10 [29]. The question whether an increased IL-10 concentration is combined with the protection against VTE in sarcoidosis, needs further investigations.

\section{Clinical characteristics}

The risk factor for the development of VTE in sarcoidosis might be related to clinical characteristics such as: compression of pulmonary arteries by the enlarged mediastinal lymph nodes. Nevertheless, according to the literature, mediastinal lymphadenopathy was combined rather with thoracic vein thrombosis than pulmonary artery thrombosis [10-12].

Patients with chronic disabling sarcoidosis on steroid therapy (resulting in immobilization because of pulmonary fibrosis, pulmonary hypertension, osteoporosis with fractures, obesity etc.) should be at the highest risk of VTE [30]. Nevertheless, our own experience indicates that sarcoidosis patients in whom VTE was recognized, recruited from a group with active stage II sarcoidosis, receiving no treatment at the time of this complication, and not disabled otherwise.

In recently recognized three cases of PE in the course of sarcoidosis we have found some similarities. All of them concerned middle-aged, non-smoking males with active sarcoidosis according to international consensus [31]. None of them had severe disabling sarcoidosis with respiratory insufficiency at the time of PE development. None of our patients suffered from pulmonary hypertension prior to PE diagnosis and no-one was on immunosuppressive treatment. No clinically significant organ involvement was seen although in one case splenomegaly and peripheral lymph nodes sarcoidosis were diagnosed. Extrinsic arterial compression at a mediastinal or hilar level was not diagnosed in pulmonary angiography. As the histopathological diagnosis was based on fine needle biopsy, it was impossible to conclude about the presence of vascular granulomata as a risk factor for local thrombosis [13, 32]. In all our cases, the VTE had favorable course with no serious late complications. In all cases we observed favorable course of pulmonary sarcoidosis with no impact on PFT and exercise ability. Regarding some similarities in the clinical picture of sarcoidosis in our set of patients, we hypothesized that there might exist a prothrombotic phenotype of sarcoidosis, probably related rather to the inflammatory process than to clinical characteristics of patients.

\section{Comorbidities}

The risk of VTE in sarcoidosis patients may be also due to comorbidities. Nevertheless, Swigris et al. found that sarcoidosis decedents with PE were even less likely than those without PE to have certain other conditions predisposing to PE, including myocardial infarction or ischemia, congestive heart failure, cardiomyopathy, cardiac dysrhythmia, sudden cardiac death, pneumonia or stroke contributing to death [5].

The other VTE risk factors may influence the development of a thrombotic event. The risk of VTE might be associated with congenital or secondary thrombophilia. Antiphospholipid antibodies - one of the diagnostic criteria for antiphospholipid syndrome (APS) - which by definition are characterized by recurrent thromboembolic events [33], were detected in $38 \%$ of sarcoidosis patients [34]. No concomitant APS in that group of sarcoidosis patients was diagnosed. The presence of antiphospholipid antibodies was associated with extra-thoracic lesions and persistence of abnormal radiological findings in sarcoidosis, and were judged by the authors to be a useful marker for prolonged disease [34]. The authors did not search for VTE occurrence in that group of sarcoidosis patients, although they should be considered at a high risk of such events. On the other hand, in numerous case reports concerning sarcoidosis complicated by VTE, the level of antiphospholipid antibodies was not examined [6-10, 13]. Thus, so far it has been impossible to conclude based on the literature whether 
the increased risk of VTE in sarcoidosis is combined with the presence of APS. In our group of patients with sarcoidosis complicated by VTE, antiphospholipid antibodies were not found by repeated testing in any of the patients.

Factor V Leiden mutation is the most common inherited form of thrombophilia [35]. Nevertheless, at least $50 \%$ of thrombotic episodes in individuals with Factor $\mathrm{V}$ Leiden mutation are provoked by additional predisposing factors [36]. To the best of our knowledge, only one case of sarcoidosis with Factor V Leiden mutation, presenting with cerebral venous thrombosis was described in the literature [9]. Authors concluded that since most factor V Leiden gene heterozygous individuals do not develop clinical thrombosis, the venous thrombosis of their patient suggested convergence of an inherited predisposition (heterozygous Factor V Leiden mutation) with an acquired thrombogenic stimulus (sarcoidosis). We have recently described a case of sarcoidosis complicated by PE in a sarcoidosis patient, in whom subsequently congenital Factor V Leiden mutation was found (data not published). We observed the favorable course of pulmonary sarcoidosis (total resolution of chest lymphadenopathy) with no impact on PFT and exercise ability during anticoagulation therapy in our patient with PE. A persistent disappearance of the pulmonary infiltrates in the patient with sarcoidosis during anticoagulant treatment for venous thrombosis has already been observed [37]. It is tempting to speculate about the anti-inflammatory potential of antithrombotic therapy. Unfractionated heparin (UFH) and LMWH proved to have the anti-inflammatory properties [38].

\section{Conclusions}

Pulmonary embolism, as a potentially fatal condition, may complicate clinically insignificant sarcoidosis. Pulmonary embolism should be considered in all patients with sarcoidosis and worsening respiratory symptoms in presence of a high plasma DD level although elevated plasma DD should be interpreted in the context of an active inflammatory process. In our opinion, an increased risk of VTE in sarcoidosis patients may depend mostly on a procoagulative phenotype related to the activity of inflammation. Congenital or acquired thrombophilia may also play a role in pathogenesis.

\section{The authors declare no conflict of interest.}

\section{References}

1. Baughman RP, Teirstein AS, Judson MA, et al. (2001): Clinical characteristics of patients in a case control study of sarcoidosis. Am J Respir Crit Care Med 164: 1885-1889.

2. Hasday JD, Bachwich PR, Lynch JP, Sitrin RG (1988): Procoagulant and plasminogen activator activities of bronchoal- veolar fluid in patients with pulmonary sarcoidosis. Exp Lung Res 14: 261-278.

3. Moller DR (1999): Cells and cytokines involved in the pathogenesis of sarcoidosis. Sarcoidosis Vasc Diffuse Lung Dis 16: 24-31.

4. Crawshaw AP, Wotton CJ, Yeates DG, et al. (2011): Evidence for association between sarcoidosis and pulmonary embolism from 35-year record linkage study. Thorax 66: 447-448.

5. Swigris JJ, Olson AL, Huie TJ, et al. (2011): Increased risk of pulmonary embolism among US decedents with sarcoidosis from 1988 to 2007. Chest 140: 1261-1266.

6. Wynne JW, Ryerson GG, Dalovisio J (1979): Myocardial sarcoidosis complicated by mural thrombosis. Thorax 34: 127-129.

7. Akova YA, Kansu T, Duman S (1993): Pseudotumor cerebri secondary to dural sinus thrombosis in neurosarcoidosis. J Clin Neuroophthalmol 13: 188-189.

8. Byrne JV, Lawton CA (1983): Meningeal sarcoidosis causing intracranial hypertension secondary to dural sinus thrombosis. Br J Radiol 56: 755-757.

9. Selvi A, Diakou M, Giannopoulos S, et al. (2009): Cerebral venous thrombosis in a patient with sarcoidosis. Intern Med 48: 723-725.

10. Marc K, Bourkadi JE, Benamor J, Iraqi G (2008): Thoracic venous thrombosis in the course of sarcoidosis. Rev Mal Respir 25: 105-106.

11. McLaughlin AM, McNicholas WT (2003): Sarcoidosis presenting as upper extremity venous thrombosis. Thorax 58: 552.

12. Rebeiz TJ, Mahfouz R, Taher A, et al. (2009): Unusual presentation of a sarcoid patient: multiple arterial and venous thrombosis with chest lymphadenopathy. J Thromb Thrombolysis 28: 245-247.

13. Moreno-Merlo F, Wanless IR, Shimamatsu K, et al. (1997): The role of granulomatous phlebitis and thrombosis in the pathogenesis of cirrhosis and portal hypertension in sarcoidosis. Hepatology 26: 554-560.

14. Reitsma PH, Rosendaal FR (2004): Activation of innate immunity in patients with venous thrombosis: the Leiden Thrombophilia Study. J Thromb Haemost 2: 619-622.

15. van Aken BE, den HM, Bos GM, et al. (2000): Recurrent venous thrombosis and markers of inflammation. Thromb Haemost 83: 536-539.

16. Mana J, Salazar A, Manresa F (1994): Clinical factors predicting persistence of activity in sarcoidosis: a multivariate analysis of 193 cases. Respiration 61: 219-225.

17. Muller-Quernheim J (1998): Serum markers for the staging of disease activity of sarcoidosis and other interstitial lung diseases of unknown etiology. Sarcoidosis Vasc Diffuse Lung Dis 15: 22-37.

18. Neville E, Walker AN, James DG (1983): Prognostic factors predicting the outcome of sarcoidosis: an analysis of 818 patients. Q J Med 52: 525-533.

19. Takizawa H, Satoh M, Okazaki H, et al. (1997): Increased IL-6 and IL-8 in bronchoalveolar lavage fluids (BALF) from patients with sarcoidosis: correlation with the clinical parameters. Clin Exp Immunol 107: 175-181.

20. Hunninghake GW, Crystal RG (1981): Mechanisms of hypergammaglobulinemia in pulmonary sarcoidosis. Site of increased antibody production and role of $\mathrm{T}$ lymphocytes. J Clin Invest 67: 86-92.

21. Miyara M, Amoura Z, Parizot C, et al. (2006): The immune paradox of sarcoidosis and regulatory T cells. J Exp Med 203: 359-370. 
22. Newman LS, Rose CS, Maier LA (1997): Sarcoidosis. N Engl J Med 336: 1224-1234.

23. Cermak J, Key NS, Bach RR, et al. (1993): C-reactive protein induces human peripheral blood monocytes to synthesize tissue factor. Blood 82: 513-520.

24. Chapman HA, Allen CL, Stone OL (1986): Abnormalities in pathways of alveolar fibrin turnover among patients with interstitial lung disease. Am Rev Respir Dis 133: 437-443.

25. Shorr AF, Thomas SJ, Alkins SA, et al. (2002): D-dimer correlates with proinflammatory cytokine levels and outcomes in critically ill patients. Chest 121: 1262-1268.

26. Baydur A, Alavy B, Nawathe A, et al. (2011): Fatigue and plasma cytokine concentrations at rest and during exercise in patients with sarcoidosis. Clin Respir J 5: 156-164.

27. Kawaguchi H, Ina Y, Sato S, et al. (1995): Serum levels of soluble receptors for tumor necrosis factor in sarcoidosis. Nihon Kyobu Shikkan Gakkai Zasshi 33: 850-855.

28. De AV, Scurati S, Raschi E, et al. (2009): Pro-inflammatory genotype as a risk factor for aPL-associated thrombosis: Report of a family with multiple anti-phospholipid positive members. J Autoimmun 32: 60-63.

29. Bingisser R, Speich R, Zollinger A, et al. (2000): Interleukin-10 secretion by alveolar macrophages and monocytes in sarcoidosis. Respiration 67: 280-286.

30. Alvarez RA, Barbash IJ, Rose JJ (2014): Bosentan for sarcoidosis-associated pulmonary hypertension, age-adjusted D-dimer levels in pulmonary embolism, and mean arterial blood pressure targets in septic shock. Am J Respir Crit Care Med 190: 948-949.

31. Costabel U, Hunninghake GW (1999): ATS/ERS/WASOG statement on sarcoidosis. Sarcoidosis Statement Committee. American Thoracic Society. European Respiratory Society. World Association for Sarcoidosis and Other Granulomatous Disorders. Eur Respir J 14: 735-737.

32. Rosen Y, Moon S, Huang CT, et al. (1977): Granulomatous pulmonary angiitis in sarcoidosis. Arch Pathol Lab Med 101: 170-174.

33. Wilson WA, Gharavi AE, Koike T, et al. (1999): International consensus statement on preliminary classification criteria for definite antiphospholipid syndrome: report of an international workshop. Arthritis Rheum 42: 1309-1311.

34. Ina Y, Takada K, Yamamoto M, et al. (1994): Antiphospholipid antibodies. A prognostic factor in sarcoidosis? Chest 105: 1179-1183.

35. Kujovich JL (2011): Factor V Leiden thrombophilia. Genet Med 13: 1-16.

36. Lensen RP, Bertina RM, de RH, Vandenbroucke JP, Rosendaal FR (2000): Venous thrombotic risk in family members of unselected individuals with factor V Leiden. Thromb Haemost 83: 817-821.

37. Hedfors E (1977): Anticoagulant treatment in sarcoidosis. Acta Med Scand 202: 237-240.

38. Downing LJ, Strieter RM, Kadell AM, et al. (1998): Lowdose low-molecular-weight heparin is anti-inflammatory during venous thrombosis. J Vasc Surg 28: 848-854. 